\title{
Diagnósticos de enfermagem mais utilizados em um hospital de urgência e emergência considerando a taxonomia da NANDA
}

\author{
Nursing diagnoses most used in an urgency and emergency hospital considering the \\ NANDA taxonomy
}
Diagnósticos de enfermería más utilizados en un hospital de urgencia y emergencia considerando la taxonomía NANDA

Cassia de Oliveira Pinto Rosa ${ }^{1 *}$, Poliana Deyse Pereira Gouvêa ${ }^{1}$, Tatiane Maestá ${ }^{1}$, Angelica Inacio da Cruz Oliveira ${ }^{1}$, Emanoela Maria Rodrigues de Sousa ${ }^{1}$, Bianca Gabriela da Rocha Ernandes ${ }^{2}$, Cassia Lopes de Sousa ${ }^{2}$, Sara Dantas ${ }^{2}$, Wuelison Lelis de Oliveira².

\section{RESUMO}

Objetivo: Identificar os diagnósticos de enfermagem frente as principais patologias observadas em um setor de urgência e emergência, entre os anos de 2017 a 2018, assim como, a importância da utilização da SAE como instrumento principal do enfermeiro ao paciente atendido no setor de Urgência e Emergência. Métodos: Trata-se de um estudo exploratório qualitativo, descritivo do tipo retrospectivo com investigação documental dos atendimentos realizados e após a elaboração de um quadro com os principais diagnósticos de enfermagem frente as patologias adquiridas. Resultados: Os dados foram fornecidos pelo Departamento de Informática do Sistema Único de Saúde (DATASUS), disponibilizado no TABNET, selecionando as principais patologias que foram atendidas no setor de urgência e emergência, e definindo os principais diagnósticos de enfermagem utilizando-se a NANDA 2018-2020 como guia para a seleção de diagnósticos e intervenções. Conclusão: Obteve-se 51 diagnósticos de enfermagem no total, os de maior prevalência foram: Risco de integridade da pele prejudicada e risco de desequilíbrio eletrolítico correspondendo respectivamente a 5,88\%, representando um total de $11,76 \%$.

Palavras-chave: Urgência, Emergência, Enfermagem, Sistematização assistência enfermagem (SAE).

\begin{abstract}
Objective: To identify nursing diagnoses in the face of the main pathologies observed in an urgency and emergency sector, between the years 2017 to 2018, as well as the importance of using SAE as the main instrument of the nurse to the patient attended in the Urgency and Emergency. Methods: This is an exploratory qualitative, descriptive retrospective study with documentary investigation of the care provided and after the elaboration of a table with the main nursing diagnoses in the face of acquired pathologies. Results: Data were provided by the Informatics Department of the Unified Health System (DATASUS), available on TABNET, selecting the main pathologies that were attended to in the urgency and emergency sector, and defining the main nursing diagnoses using NANDA 2018-2020 as a guide for selecting diagnoses and interventions. Conclusion: 51 nursing diagnoses were obtained in total, the most prevalent were: Risk of impaired skin integrity and risk of electrolyte imbalance corresponding to $5.88 \%$, respectively, representing a total of $11.76 \%$.
\end{abstract}

Keywords: Urgency, Emergency, Nursing, Nursing care systematization (SAE).

${ }^{1}$ Complexo Hospitalar Regional de Cacoal (COHREC), Cacoal - RO.

*E-mail: cassiaenfermagem@outlook.com

2 Faculdade de Ciências Biológicas de Cacoal (Facimed), Cacoal - RO.

SUBMETIDO EM: 9/2020

ACEITO EM: 10/2020

PUBLICADO EM: 2/2021 


\section{RESUMEN}

Objetivo: Identificar los diagnósticos de enfermería ante las principales patologías observadas en un sector de urgencia y emergencia, entre los años 2017 a 2018, así como la importancia de utilizar el SAE como principal instrumento de la enfermera al paciente atendido en la Urgencia y Emergencia. Métodos: Se trata de un estudio exploratorio, cualitativo, descriptivo retrospectivo con investigación documental del cuidado brindado y posterior a la elaboración de una tabla con los principales diagnósticos de enfermería ante patologías adquiridas. Resultados: Los datos fueron proporcionados por el Departamento de Informática del Sistema Único de Salud (DATASUS), disponible en TABNET, seleccionando las principales patologías atendidas en el sector de urgencia y emergencia, y definiendo los principales diagnósticos de enfermería mediante NANDA 2018-2020 como guía para la selección de diagnósticos e intervenciones. Conclusión: Se obtuvieron 51 diagnósticos de enfermería en total, los más prevalentes fueron: Riesgo de deterioro de la integridad cutánea y riesgo de desequilibrio electrolítico correspondientes al 5,88\%, respectivamente, lo que representa un total de $11,76 \%$.

Palabras clave: Urgencia, Emergencia, Enfermería, Sistematización de la atención de enfermería (SAE).

\section{INTRODUÇÃO}

O país enfrenta múltiplos problemas com acrescente demanda nos serviços de urgência, como dimensionamento de pessoal insuficiente com sobrecarga de trabalho manual, superlotação de leitos e pouca articulação referente as redes assistenciais. Entretanto, com todas estas contrariedades, é primordial prestar uma assistência contínua e de qualidade aos usuários do serviço, requerendo, assim, processos de trabalho bem esclarecidos e profissionais de saúde altamente capacitados (MIRANDA CA, et al., 2012).

Diante dos vários requisitos adversos que rodeiam o campo dos serviços de urgência e emergência, a atuação do enfermeiro se torna substancial junto à equipe multidisciplinar, tendo em vista a Sistematização da Assistência de Enfermagem (SAE) como uma considerável ferramenta de gerenciamento do processo de cuidado de cada usuário. Embora seja bem definida e respaldada por aspectos legais e científicos, a SAE ainda é pouco presente nesse tipo de serviço de saúde (FELIX NN, et al., 2009; e MIRANDA CA, et al., 2012).

O Conselho Federal de Enfermagem (COFEN), através das Resoluções no 375/2011, regulamenta o trabalho dos enfermeiros nas urgências e emergências, no atendimento Pré-Hospitalar e Inter Hospitalar, em situações de risco conhecido ou desconhecido (COFEN, 2011).

O setor de emergência hospitalar pode ser considerado como uma das áreas de maior complexidade da assistência, devido ter maior fluxo de atividades de profissionais e necessidades dos usuários. Uma área que exige uma assistência imediata, eficiente e de amplo conhecimento técnico, habilidade profissional e o emprego de recursos tecnológicos. A enfermagem é uma ciência que se aperfeiçoa constantemente e a Sistematização da Assistência de Enfermagem (SAE) possibilita que o enfermeiro desenvolva e aplique seus conhecimentos visando uma assistência de qualidade (SOUZA NR, et al., 2015).

A Resolução COFEN no 358/2009 nos diz que a SAE é uma atividade privativa do enfermeiro, sendo uma prática científica que contribui junto com as ações de enfermagem para promoção, prevenção, recuperação e reabilitação da saúde do indivíduo, família ou comunidade (CONFEN, 2009).

Ainda segundo a resolução acima, a SAE tem como alvo reduzir as complicações durante o tratamento, facilitar a recuperação e adaptação do paciente, família e comunidade, promovendo sua saúde e o seu bemestar, sendo composta por cinco etapas: Coleta de dados e investigação; Diagnóstico de enfermagem; Planejamento da assistência; Implementação da assistência e Avaliação dos resultados (CONFEN, 2009).

É preciso que ocorra algumas mudanças para que a enfermagem se fortaleça cientificamente. Para tanto, o enfermeiro necessita planejar, refletir e justificar suas intervenções, a partir da Sistematização da Assistência de Enfermagem (SAE) (MARIA MA, et al., 2012). 
De maneira geral, os enfermeiros conhecem a SAE, alguns possuem uma visão limitada e restrita, porém, tem outros que compreendem a SAE de maneira ampla e a consideram um instrumento de organização que facilita a assistência. Os que possuem uma visão simplificada e não a utilizam, precisam progredir quanto à sua implantação e implementação, pois a SAE é o método de organizar a assistência a ser prestada ao cliente, família e comunidade, permitindo a construção de um saber próprio e autônomo (VARELA GC e FERNANDES SCA, 2013).

Além de fornecer subsídios para a atuação com maior segurança e estímulo à pesquisa, estudar as causas e as consequências de uma doença é fundamental, a fim de estabelecer diagnóstico e contribuir para a adoção de medidas de prevenção, controle, assistência e educação (CALIL AM e PIMENTA CAM, 2010).

O Processo de Enfermagem (PE) se divide em cinco etapas inter-relacionadas e interdependentes, sendo: coleta de dados de enfermagem, diagnóstico de enfermagem, planejamento de enfermagem, implementação e avaliação de enfermagem (COFEN, 2009). No que se refere à segunda etapa, o processo de se estabelecer diagnósticos exige raciocínio clínico e conhecimento da taxonomia, uma vez que esta definição deve se aplicar à realidade observada (FRANCO B, 2015).

Sendo a segunda etapa do processo, os diagnósticos de enfermagem são examinados criteriosamente e interpretados para identificar os problemas reais, quanto os problemas potenciais, sendo elaborados de acordo com os protocolos da instituição, os mais trabalhados são National Conference of the Nursing American Diagnosis Association (NANDA) e Classificação Internacional para a Prática de Enfermagem (CIPE).

Os Diagnósticos de Enfermagem (DE) são julgamentos clínicos sobre as respostas do indivíduo, da família ou da comunidade a problemas de saúde. Contribuindo para uma prática de assistência qualificada, uma vez que determina o foco da prática clínica, é a base para o planejamento das intervenções de enfermagem, principalmente junto a vítimas graves e de maior complexidade (GALDEANO LE e ROSSI LA, 2002; CARVALHO EC, 2008).

Considerando a responsabilidade do enfermeiro junto aos pacientes graves e críticos, à formulação de diagnósticos de enfermagem fundamentada na identificação de sinais e sintomas, à rapidez no raciocínio clínico e às especificidades do atendimento a essas vítimas, o presente estudo teve como objetivo identificar os diagnósticos de enfermagem frente as principais patologias observadas em um setor de urgência e emergência.

\section{MÉTODOS}

Trata-se de um estudo exploratório qualitativo, descritivo do tipo retrospectivo com investigação documental dos atendimentos realizados em um hospital de ensino no interior de Rondônia através da Central de Regulação de Urgência e Emergência (CRUE) que é responsável pela regulação de pacientes em 32 municípios.

A coleta foi realizada através de análise dos atendimentos e dados fornecidos pelo Departamento de Informática do Sistema Único de Saúde (DATASUS), disponibilizado no TABNET onde o período da amostra recolhida foi do ano de 2017 ao ano de 2018. E diante da análise documental selecionando as principais patologias atendidas no setor de urgência e emergência, foi definido os principais diagnósticos de enfermagem utilizando-se a NANDA 2018-2020 como guia para a seleção de diagnósticos.

O estudo foi aprovado pelo Comitê de Ética e Pesquisa (CEP) da Faculdade de Ciências Biomédicas de Cacoal (FACIMED), via Plataforma Brasil, com o número CAAE: 97260718.5.0000.5298 e parecer oㅡ 2.887.277.

\section{RESULTADO E DISCUSSÃO}

A Resolução do COFEN 311/2007, art. 12 a 14, nos diz que o profissional de enfermagem é responsável em prestar uma assistência com competência técnica, cientifica e ética, livre de danos para o indivíduo 
decorridos de imperícia, negligência ou imprudência, bem como aprimorar seus conhecimentos em benefício à pessoa e ao desenvolvimento da profissão. Desta maneira, é de suma importância refletir sobre a elaboração e planejamento da assistência de enfermagem que deve ser prestado ao paciente, principalmente no setor de urgência e emergência (CONFEN, 2007).

Diante da análise documental, podemos observar com o Tabela 1, os atendimentos que mais foram realizados na sala vermelha, e com base nisso foi definido os principais diagnósticos e intervenções de enfermagem de cada patologia, como pode ser analisado no Quadro 1.

Tabela 1 - Principais patologias atendidas na sala vermelha nos anos de 2017-2018.

\begin{tabular}{cccc}
\hline Diagnósticos clínicos & $\mathbf{2 0 1 7}$ & $\mathbf{2 0 1 8}$ & Total \\
\hline Tratamento Oncológico & 815 & 877 & 1.692 \\
Traumatismos & 542 & 628 & 1.170 \\
AVE- H e AVE- I & 307 & 298 & 605 \\
Pneumonias Virais E Influenzas & 229 & 280 & 509 \\
Insuficiência Cardíaca Congestiva (ICC) & 223 & 236 & 459 \\
Infarto Agudo Miocárdio (IAM) & 204 & 222 & 426 \\
Traumatismo Crânio Encefálico & 219 & 140 & 359 \\
Diabetes Melittus (DM) & 164 & 190 & 354 \\
Apendicectomia & 129 & 156 & 285 \\
Laparotomia Exploratória & 65 & 36 & 101 \\
Intoxicações Exógenas & 45 & 35 & 80 \\
\hline
\end{tabular}

Fonte: Rosa COP, et al., 2020. Baseado em dados do DATASUS de 2017 a 2018.

Analisando os dados encontrados no Quadro 1, as principais patologias atendidas na sala vermelha nos anos de 2017 à 2018, da amostra composta por 6.040 pacientes atendidos no período do estudo, foram de atendimentos ao paciente em tratamento oncológico com total de $1.692(28,01 \%)$ dos casos; traumatismos 1.170 (17,37\%); AVE-H e AVE-I 605 (10,01\%); pneumonias virais e influenzas 509 (9,76\%); insuficiência cardíaca congestiva 459 (7,59\%); infarto agudo do miocárdio 426 (7,05\%); traumatismo crânio encefálico 359 (5,94\%); diabetes mellitus 354 (5,86\%); apendicectomia 285 (4,71\%); laparotomia exploratória 101 (1,67\%) e intoxicações exógenas com 80 casos, correspondendo a 1,32\%. Jáno quadro abaixo observa-se os principais rótulos diagnósticos de enfermagem aplicados a essas patologias. 
Quadro 1 - Principais rótulos diagnósticos das principais patologias atendidas na sala vermelha com base na taxonomia da NANDA Internacional (2018).

\begin{tabular}{|c|c|}
\hline Patologias & Rótulos diagnóstico de enfermagem \\
\hline Tratamento Oncológico & $\begin{array}{c}\text { Dor crônica } \\
\text { Medo } \\
\text { Náusea } \\
\text { Nutrição desequilibrada: menos que do que as } \\
\text { necessidades corporais } \\
\text { Risco de integridade da pele prejudicada }\end{array}$ \\
\hline Traumatismo crânio encefálico & $\begin{array}{c}\text { Capacidade adaptativa intracraniana diminuída } \\
\text { Termorregulação ineficaz } \\
\text { Integridade tissular periférica prejudicada } \\
\text { Risco de perfusão tissular cerebral ineficaz } \\
\text { Risco de integridade da pele prejudicada } \\
\end{array}$ \\
\hline Acidente vascular encefálico & $\begin{array}{c}\text { Comunicação verbal prejudicada } \\
\text { Risco de perfusão tissular cerebral ineficaz } \\
\text { Risco de confusão aguda } \\
\text { Risco de Disfunção neurovascular periférica } \\
\text { Risco de integridade da pele prejudicada }\end{array}$ \\
\hline Infarto agudo do miocárdio & $\begin{array}{c}\text { Ansiedade } \\
\text { Dor aguda } \\
\text { Débito cardíaco diminuído } \\
\text { Intolerância à atividade } \\
\text { Risco de perfusão tissular cardíaca diminuída }\end{array}$ \\
\hline Insuficiência cardíaca & $\begin{array}{c}\text { Débito cardíaco diminuído } \\
\text { Volume de líquidos excessivo } \\
\text { Fadiga } \\
\text { Intolerância à atividade } \\
\text { Risco de perfusão tissular periférica ineficaz }\end{array}$ \\
\hline $\begin{array}{c}\text { Doenças respiratórias agudas, } \\
\text { infecções respiratórias agudas, } \\
\text { Pneumonia }\end{array}$ & $\begin{array}{c}\text { Troca de gases prejudicada } \\
\text { Fadiga } \\
\text { Padrão respiratório ineficaz } \\
\text { Ventilação espontânea prejudicada } \\
\text { Risco de perfusão tissular periférica ineficaz }\end{array}$ \\
\hline Intoxicação exógena & $\begin{array}{c}\text { Medo } \\
\text { Ansiedade relacionada à morte } \\
\text { Risco de desequilíbrio eletrolítico } \\
\text { Risco de síndrome do desequilíbrio metabólico }\end{array}$ \\
\hline Trauma abdominal & $\begin{array}{c}\text { Risco de motilidade gastrintestinal disfuncional } \\
\text { Risco de choque } \\
\text { Dor aguda } \\
\text { Risco de sangramento } \\
\text { Risco de recuperação cirúrgica retardada }\end{array}$ \\
\hline Trauma ortopédico & $\begin{array}{c}\text { Mobilidade física prejudicada } \\
\text { Risco de Disfunção neuro- vascular periférica } \\
\text { Risco de tromboembolismo venoso } \\
\text { Conforto prejudicado } \\
\text { Integridade tissular periférica prejudicada }\end{array}$ \\
\hline Diabetes descompensada & $\begin{array}{c}\text { Risco de glicemia instável } \\
\text { Risco de desequilíbrio eletrolítico } \\
\text { Risco de síndrome do desequilíbrio metabólico }\end{array}$ \\
\hline Insuficiência renal aguda & $\begin{array}{c}\text { Eliminação urinária prejudicada } \\
\text { Volume de líquidos excessivo } \\
\text { Risco de desequilíbrio eletrolítico } \\
\text { Risco de volume de líquidos desequilibrado }\end{array}$ \\
\hline
\end{tabular}

Fonte: Rosa COP, et al., 2020. 
Diante da análise do Quadro 1, as intervenções de enfermagem citadas a seguir foram recomendações realizadas pelas autoras enfermeiras, com base nos diagnósticos que foram identificados e de acordo com a Classificação das Intervenções de Enfermagem (NIC), levando, portanto, em referência as características definidoras e os fatores relacionados de cada patologia da sala vermelha e os fatores de riscos para subsequentemente serem analisado cada medida a ser adotada pela equipe de enfermagem.

Com isso, discutiu-se as principais intervenções de acordo com cada patologia e diagnóstico de enfermagem, notou-se a predominância de diversas intervenções, tais como: Instalar $\mathrm{O}_{2}$ se saturação $<90 \%$ correspondendo $11,11 \%$ das medidas de cuidados prestados aos pacientes, em seguida monitorar saturação de $\mathrm{O}_{2} \mathrm{com} 10 \%$, realizar aquecimento de extremidades (6,66\%); realizar balanço hídrico (5,55\%); proporcionar conforto ao paciente, realizar mudança de decúbito de $2 / 2 \mathrm{~h}$, cuidados com a pele, instalar e mensurar PVC e avaliar a gasometria arterial rigorosa (4,44\%); monitorar e avaliar nível de dor e avaliar rigorosamente o nível de consciência por meio da escala de coma de Glasgow (3,33\%); monitorar capacidade de deglutir, Realizar exercícios de alongamento, criar estratégias que reduzem a ansiedade, implementar apoio emocional, administrar medicação conforme prescrição médica, realizar ECG de rotina $(2,22 \%)$ dentre inúmeras outras, cada uma delas, proposta aos diagnósticos de enfermagem, referente as patologias citadas anteriormente conforme mostra o Quadro 1.

No Tratamento Oncológico pode-se ter como intervenções segundo a taxonomia NANDA Internacional (2018) o monitoramento cabendo ao enfermeiro e avaliar nível de dor, administrar medicação conforme prescrição médica, proporcionar conforto ao paciente, oferecer apoio emocional ao paciente, incentivar ingesta hídrica e alimentar, realizar mudança de decúbito de $2 / 2 \mathrm{~h}$ e mantendo os cuidados necessários com a pele destes pacientes.

Já no traumatismo crânio encefálico identifica-se como algumas intervenções a anotação e mensuração do PIC, o enfermeiro deve avaliar, rigorosamente, o nível de consciência por meio da escala de coma de Glasgow, monitorar capacidade de deglutir, usar manta térmica, realizar compressas frias ou mornas, realizar aquecimento de extremidades, monitorar saturação de $\mathrm{O}_{2}$, instalar $\mathrm{O}_{2}$ se saturação $<90 \%$, realizar mudança de decúbito de $2 / 2 \mathrm{~h}$ e cuidados com a pele.

Diante o acidente vascular encefálico considera-se proporcionar como cuidados: métodos que facilite a comunicação da paciente, avaliar rigorosamente o nível de consciência por meio da escala de coma de Glasgow, monitorar capacidade de deglutir, realizar aquecimento de extremidades, realizar exercícios de alongamento, monitorar saturação de $\mathrm{O}_{2}$, instalarO $\mathrm{O}_{2}$ se saturação $<90 \%$, realizar mudança de decúbito de 2/2h e cuidados com a pele.

Quanto as demais principais patologias encontradas, nota-se algumas propostas de intervenções para cada diagnóstico de enfermagem, para Infarto Agudo do Miocárdio (IAM): criar estratégias que reduzem a ansiedade, implementar apoio emocional, monitorar e avaliar nível de dor, administrar medicação conforme prescrição médica, realizar ECG de rotina, realizar balanço hídrico, repouso absoluto, minimizar esforço físico, monitorar saturação de $\mathrm{O}_{2}$, instalar $\mathrm{O}_{2}$ se saturação $<90 \%$ e identificar sinais de alarme.

Insuficiência cardíaca: realizar ECG de rotina, realizar balanço hídrico, instalar e mensurar PVC, instalar e mensurar PAI, ingesta hídrica rigorosa, monitorar saturação de $\mathrm{O}_{2}$, instalar $\mathrm{O}_{2}$ se saturação $<90 \%$, repouso absoluto, minimizar esforço físico e realização de aquecimento de extremidades.

Doenças respiratórias agudas, infecções respiratórias agudas, pneumonia: monitorar saturação de Ozinstalar $\mathrm{O}_{2}$ se saturação $<90 \%$, elevar cabeceira no mínimo $45^{\circ}$ grau, realizar aquecimento de extremidades, avaliar a gasometria arterial rigorosa e proporcionar conforto ao paciente.

Intoxicação exógena: criar estratégias que reduzem a ansiedade, implementar apoio emocional, realizar balanço hídrico, instalar e mensurar PVC, avaliar a gasometria arterial rigorosa, monitorar, saturação de $\mathrm{O}_{2}$, instalar $\mathrm{O}_{2}$ se saturação $<90 \%$.

No trauma abdominal identifica-se como as intervenções mais apropriadas diante esta patologia a realização e anotação do débitos das SNG, deve-se, realizar ausculta antes das administrações de 
medicamentos e alimentação, realizar controle rigoroso de sinais como debito urinário, pressão venosa central e lactato, monitorização rigorosa dos sinais vitais, monitorar e avaliar nível de dor, administrar medicação conforme prescrição médica, avaliar, rigorosamente, o nível de consciência por meio da escala de coma de Glasgow, instalar $\mathrm{O}_{2}$ se saturação $<90 \%$, realizar aquecimento de extremidades e proporcionar conforto ao paciente. No trauma ortopédico o enfermeiro necessita realizar exercícios de alongamento, realizar mudança de decúbito de $2 / 2 \mathrm{~h}$, realiza cuidados com a pele do paciente, monitorar saturação de $\mathrm{O}_{2}$, instalar $\mathrm{O}_{2}$ se, saturação $<90 \%$, proporcionar conforto ao paciente e realizar aquecimento de extremidades.

Diabetes descompensada: Realizar glicemia capilar de 4/4h, realizar balanço hídrico, instalar e mensurar PVC, avaliar a gasometria arterial rigorosa, monitorar saturação de $\mathrm{O}_{2}$ e instalar $\mathrm{O}_{2}$ se saturação $<90 \%$. $\mathrm{Na}$ insuficiência renal aguda algumas intervenções e condutas de enfermagem que mantém a assistência e o cuidado: realizar balanço hídrico, ingesta hídrica rigorosa, instalar e mensurar PVC, anotar e mensurar, peso diariamente, avaliar a gasometria arterial rigorosa, monitorar saturação de $\mathrm{O}_{2}$ e instalar $\mathrm{O}_{2}$ se saturação $<90 \%$.

O enfermeiro em âmbito hospitalar desenvolve inúmeras tarefas com alto grau de exigências e responsabilidades. O cuidado ao paciente grave, nos setores de emergência, demanda dos enfermeiros organização, registros adequados e conhecimento clínico, mediante a complexidade do quadro em que um paciente criticamente enfermo se encontra (SOARES MI, et al.,2015; SANTOS SM, et al.,2018).

No Quadro 1, também se destacaram os principais diagnósticos de enfermagem aplicados as principais patologias atendidas na sala vermelha de acordo com a taxonomia da NANDA Internacional. Obteve-se 51 diagnósticos de enfermagem no total, os de maior prevalência foram: Risco de integridade da pele prejudicada e risco de desequilíbrio eletrolítico correspondendo respectivamente a 5,88\%, representando um total de $11,76 \%$, sendo identificados nas seguintes patologias, tratamento oncológico, Traumatismo Crânio Encefálico (TCE), Acidente Vascular Encefálico (AVE), intoxicação exógena, diabetes descompensada e insuficiência renal aguda.

O diagnóstico de enfermagem de "Risco de infecção" não foi citado no quadro, devido todos as patologias compreender esse diagnóstico, pois o paciente internado em um hospital apresenta exposição ambiental a patógenos, principalmente em uma unidade de emergência, onde muitos procedimentos invasivos são normalmente realizado.

Os diagnósticos medo, risco de integridade da pele prejudicada, integridade tissular periférica prejudicada, risco de perfusão tissular cerebral ineficaz, risco de disfunção neuro-vascular periférica, dor aguda, débito cardíaco diminuído, intolerância à atividade, volume de líquido excessivo, fadiga, risco de desiquilíbrio eletrolítico e risco de perfusão tissular periférica ineficaz corresponderam à 3,92\% nesta ordem, sendo o total de $35,28 \%$, tais diagnósticos foram atribuídos as seguintes patologias tratamento oncológico, intoxicação exógena, TCE, AVE, Infarto Agudo do Miocárdio (IAM), Insuficiência Cardíaca (IC), doenças respiratórias agudas, infecções respiratórias agudas/pneumonia, trauma abdominal, trauma ortopédico, diabetes descompensada e insuficiência renal aguda. Os demais diagnósticos não citados anteriormente presentes na tabela 2 corresponderam a um total de $52,96 \%$.

A qualidade na assistência ao paciente depende da conduta da equipe de saúde, em especial dos enfermeiros na aplicação da Sistematização da Assistência em Enfermagem (SAE). Em um estudo realizado com enfermeiros demonstrou-se que os profissionais não compreendiam sobre a sistematização, $70 \%$ dos enfermeiros na pesquisa não citou nenhum diagnóstico de enfermagem e não os utilizavam durante a sua prática profissional, é considerado que a equipe de enfermagem é a que está em contato direto ao paciente, se tornando de grande importância o conhecimento da aplicação da SAE (SILVA ECG, et al., 2011).

$\mathrm{Na}$ enfermagem se utiliza muito a SAE para o direcionamento do cuidado a qual será prestado, para que haja atribuição da segurança do paciente e dos profissionais esta sistematização proporciona ao enfermeiro a identificação das necessidades do cliente e conduz toda equipe durante as intervenções que serão realizadas, concedendo ao enfermeiro o desenvolvimento de suas habilidades e permitindo que este profissional avalie na tomada decisões sobre a assistência que será prestada ao paciente, determinando todos os critérios para resultados satisfatórios (MENEZES SRT, et al., 2011). 
O uso da Sistematização da Assistência de Enfermagem (SAE) busca o pensamento crítico do enfermeiro, auxiliando-o no alcance de resultados satisfatórios e nos cuidados com o propósito de amparar a recuperação do paciente, assim o profissional de enfermagem deve ter habilidade diante da SAE, seguindo as medidas de conduta correta e tendo ética profissional. A aplicação da sistematização circunda etapas a serem seguidas pelo enfermeiro, exercendo a autonomia fundamentada nos conhecimentos técnico-científicos, bem como a familiaridade com cada diagnóstico de enfermagem e compreensão da adaptação das necessidades básicas de cada paciente perante o seu diagnóstico (SILVA EGC, et al., 2011).

No estudo realizado por Santos SM, et al., (2018), as enfermeiras relataram uma problemática entre implementar a SAE e realizar as demais atividades essenciais ao cuidado do paciente crítico, que por muitas vezes é complexo e exige elevada carga de trabalho da enfermagem, citando também fatores como o dimensionamento inadequado de pessoal e o absenteísmo, que pode refletir diretamente no Processo de Enfermagem.

O presente estudo demonstrou que no período de 2017 e 2018 na sala vermelha foram atendidas 11 patologias com diferentes diagnósticos de enfermagem, nesses anos obteve-se o resultado de 51 diagnósticos no total, dentre eles alguns se sobre-excederam. O setor de urgência e emergência tem grande fluxo de pacientes, pode-se observar que o diagnóstico de risco de integridade da pele prejudicada esteve presente em 3 doenças citadas, esse diagnóstico na maioria das vezes se relaciona a pessoas idosas, acamadas, pacientes em altas doses de sedativos, e em geral em indivíduos com mobilidade prejudicada, normalmente nos pacientes em Unidade de Terapia Intensiva (UTI) e internação prolongada são os mais afetados (CARDOSO SRS e CRUZ ICF, 2017).

Ao examinar os diagnósticos nesse estudo, observou-se que o de risco de desequilíbrio eletrolítico se destacou dentre os demais, frequentemente ocorrendo em pacientes com a homeostasia descontrolada com perca de líquido excessivo e eletrólitos, um exemplo comum em pacientes com poliúria, êmese, diarreias e o organismo como um todo também influência no controle da homeostasia, sendo necessária a troca de nutrientes para que não ocorra desequilíbrio eletrolítico (DEBONE MC, et al., 2017).

O conhecimento sobre SAE é a ferramenta principal para o cuidado ao paciente, a implementação do diagnóstico de enfermagem deve ser efetuada pelo enfermeiro posteriormente criando protocolo de avaliação para contribuir na assistência. A SAE vem sendo amplamente utilizada nos últimos anos como método científico para instrumentalizar a resolução de problemas dos pacientes e tornar o cuidado individualizado, além de embasar e fundamentar cientificamente as ações do mesmo. A criação de protocolos no Serviço de Urgência e Emergência com base nos diagnósticos de enfermagem pode direcionar o julgamento clínico do enfermeiro sobre os problemas de saúde enfrentados pelo paciente crítico, permitindo a agilidade para a elaboração de um plano de cuidado integral (SALGADO PO, et al., 2013; DUTRA CSK, et al., 2014).

\section{CONSIDERAÇÕES FINAIS}

Esta pesquisa possibilitou observar as principais patologias atendidas na sala vermelha nos anos de 20172018, identificando os 51 diagnósticos e as intervenções de enfermagem aplicadas com base na taxonomia da NANDA Internacional (2018), os mais frequentes foram: risco de integridade da pele prejudicada e risco de desequilíbrio eletrolítico. Os diagnósticos de enfermagem contribuem na elaboração de um instrumento de coleta de dados para pacientes atendidos na sala vermelha, auxiliam os profissionais nas intervenções baseando-se em evidências, com o propósito de atender as necessidades humanas básicas, bem como favorecer no processo de evolução do tratamento do paciente, contribuindo no desenvolvimento de técnicas e estratégias interdisciplinares e humanizadas de cuidado.

\section{REFERÊNCIAS}

1. CALIL AM, PIMENTA CAM. A importância da avaliação e padronização analgésica em serviços de emergência. Acta Paul Enferm. 2010;65(1):132-8. 
2. CARVALHO EC. Relações entre a coleta de dados, diagnóstico e prescrição de enfermagem a pacientes adultos de uma UTI. Rev. Latino-Am. Enfermagem. 2008;16(4):700-6

3. CARDOSO SRS, CRUZ ICF. Risco de Integridade da pele prejudicada- revisão sistematizada da literatura. Journal of Specialized Nuring Care, 2017; 9 (1).

4. CONSELHO FEDERAL DE ENFERMAGEM (Brasil). Resolução COFEN no 311 de 08 de fevereiro de 2007. Aprova a Reformulação do Código de Ética dos Profissionais de Enfermagem. Disponível em: http://www.cofen.gov.br/resoluo-cofen-3112007 4345.html. Acesso em: 28/07/2020

5. CONSELHO FEDERAL DE ENFERMAGEM (BR). Resolução no 375de 2011. Dispõe sobre a presença do Enfermeiro no Atendimento Pré-hospitalar e Inter-Hospitalar, em situações de risco conhecido ou desconhecido.

6. CONSELHO FEDERAL DE ENFERMAGEM. Resolução COFEN nํ358, de 15 de outubro de 2009. Dispõe sobre a Sistematização da Assistência de Enfermagem e a implementação do Processo de Enfermagem em ambientes, públicos ou privados, em que ocorre o cuidado profissional de Enfermagem, e dá outras providências. 2016.

7. CONSELHO FEDERAL DE ENFERMAGEM (COFEN). Resolução № 358, de 15 de outubro de 2009. Dispõe sobre a Sistematização da Assistência de Enfermagem e a implementação do Processo de Enfermagem em ambientes, públicos ou privados, em que ocorre o cuidado profissional de Enfermagem e dá outras providências.

8. DEBONE MC, et al. Diagnósticos de enfermagem em idosos com doença renal crônica em hemodiálise. Revista Brasileira de Enfermagem. 2017; 70(4): 800-805.

9. DUTRA CSK, et al. Diagnósticos de enfermagem prevalentes no paciente internado com sepse no Centro de Terapia Intensiva. Cogitare Enferm. 2014;19(4):747-54

10. FELIX NN, et al. Desafios encontrados na realização da sistematização da assistência de enfermagem (SAE) em unidade de pronto atendimento. Arq Ciência Saúde, Paraná, 16 (4): 155 -60, 2009.

11. FRANCO, B. Análise do Sistema de Triagem Manchester como subsídio para o 132 diagnóstico de enfermagem. Dissertação de Mestrado. Programa de Pós-graduação em Enfermagem, Universidade Federal do Rio Grande do Sul, 2015.

12. GALDEANO LE, ROSSI LA. Construção e validação de instrumento de coleta de dados para o período perioperatório de cirurgia cardíaca. Rev. Latino-Am. Enfermagem. 2002;10(6):800-4.

13. MARIA MA, et al. Sistematização da assistência de enfermagem em serviços de urgência e emergência: viabilidade de implantação. Rev. bras. Enferm, 2012; 65: 297-303.

14. MENEZES SRT, et al. Autonomia e vulnerabilidade do enfermeiro na prática da Sistematização da Assistência de Enfermagem. Revista da Escola de Enfermagem da USP, 2011; 45(4): 953-958.

15. MIRANDA CA, et al. Opinião de enfermeiros sobre instrumentos de atendimento sistematizado a paciente em emergência. Revista Rene, Ceará, 13 (2): 396-407, 2012.

16. SALGADO PO, et al. Diagnósticos de enfermagem em pacientes numa unidade de emergência. Rev enferm. UFPE online $-2013 ; 7(1): 83-9$.

17. SANTOS SM, et al. Desafios e potencialidades na implementação da sistematização da assistência de enfermagem a pacientes críticos. ReTEP [Internet] 2018, 10(3):9-13.

18. SILVA EGC, et al. Conhecimento do enfermeiro sobre a Sistematização da Assistência de Enfermagem: da teoria à prática. Revista da Escola de Enfermagem da USP, 2011; 45 (6): 1380-1386.

19. SOUZA NR, et al. Sistematização da Assistência de Enfermagem: dificuldades referidas por enfermeiros de um hospital universitário. Revista de enfermagem UFPR, 2015; 3(9): 7104-10.

20. SOARES MI, et al. Sistematização da assistência de enfermagem: facilidades e desafios do enfermeiro na gerência da assistência. Esc Anna Nery. 2015;19(1):47-53.

21. VARELA GC, FERNANDES SCA; Conhecimentos e práticas sobre Sistematização da Assistência de Enfermagem na estratégia saúde da família. Cogitare Enferm, 2013; 1(18): 124-30. 